\title{
Is Second Look Laparoscopy for Diagnosing Remaining Ovarian Cancer Appropriate Further Treatment?
}

\author{
Rama Inamdar*, Ahyoung Cho*, Hae Kyung Yoo, Hye-Sung Moon \\ Department of Obstetrics and Gynecology, Ewha Women Cancer Center, Ewha Womans University College of Medicine, Seoul, Korea
}

Objectives: The aim of our study is to compare the findings of investigative modalities and second look laparoscopy in ovarian cancer and establish the safety and accuracy of second look laparoscopy for detecting ovarian cancer.

Methods: We retrospectively reviewed 11 patients with ovarian cancer treated by a single surgeon from 2006 to 2013 . These patients were diagnosed at the time of primary cytoreductive surgery and received six cycles of combination chemotherapy. Then, they underwent second look laparoscopy. They were followed up with tumor markers monthly and PET-CT and/or CT scans.

Results: All 11 patients had undergone primary surgery followed by six cycles of consolidation chemotherapy. Eight patients had positive pathologic findings on second look laparoscopy $(72.7 \%)$. The CA 125 level was higher in one patient (12.5\%). In seven patients who had positive results on second look laparoscopy, the value was well below normal limits (87.5\%). Three patients had recorded increases in fluorodeoxyglucose uptake $(37.5 \%)$. The increase in standardized uptake values in specific regions in the scans corresponded to positive biopsies from those regions. Seven patients who had positive findings on second look laparoscopy were treated with consolidation chemotherapy. The 5 -year survival rate was $66.67 \%$, and the 5 -year recurrence rate was $33.33 \%$.

Conclusion: There are limitations to the accuracy of current investigative techniques, and we must rely on clinical correlation with these modalities for each case of second look laparoscopy. It is feasible to safely perform second look laparoscopy to detect remnant ovarian cancer. (Ewha Med J 2019;42(2):15-19)

\author{
Received July 16, 2018 \\ Revised March 31, 2019 \\ Accepted April 8, 2019 \\ Corresponding author \\ Hye-Sung Moon \\ Department of Obstetrics and Gynecology, \\ Ewha Women Cancer Center, Ewha \\ Womans University College of Medicine, \\ 25 Magokdong-ro 2-gil, Gangseo-gu, Seoul \\ 07804, Korea \\ Tel: 82-2-6986-1678, Fax: 82-2-2647-9860 \\ E-mail:mhsmhs@ewha.ac.kr \\ *These two authors contributed equally to this \\ study as first author.
}

Key Words

Second look; Laparoscopy; Ovarian neoplasms

\section{Introduction}

Ovarian cancer is one of the leading causes of death in women and represents a great clinical challenge. In Korea, it is the second most common gynecologic cancer after uterine cervix cancer. The worldwide incidence of ovarian cancer is estimated to be 6.3 per 100,000 . It is higher in developed countries $(9.3 / 100,000)$ compared to other countries [1]. The incidence of ovarian cancer in Korea is 8.6/100,000, lower than the incidence in Western nations; however, it has been rising steadily over time. In 2012, there were 2167 estimated new cases of ovarian cancer in Korea [2]. In recent years, the rising incidence in Korea is similar to the incidence trends reported in other Asian countries.

The mortality rate of ovarian cancer in Korea is increasing markedly, and from 1983 to 2006, mortality increased 9.5-fold nationwide (from 0.22 per 100,000 person-years in 1983 to 2.09 per 100,000 person-years). However, a slight decrease in

This is an Open Access article distributed under the terms of the Creative Commons Attribution Non-Commercial License (http://creativecommons.org/licenses/by-nc/4.0) which permits unrestricted non-commercial use, distribution, and reproduction in any medium, provided the original work is properly cited. 
mortality rates has been reported in several Western countries, including the USA and the UK, and in some Asian countries such as China, while the mortality rate in Japan has remained stable [3]. In 2012, there were 987 deaths due to ovarian cancer in Korea.

The symptoms of ovarian cancer are nonspecific. Hence, most cases are diagnosed at advanced stages and have poor prognosis. The standard treatment for ovarian cancer consists of surgery followed by systemic chemotherapy [4]. The aim of primary surgery is confirmation of diagnosis through surgical staging and optimal debulking. Patients are then regularly followed up with clinical examinations and various investigative modalities for any recurrence. For cases of advanced ovarian cancer, second look surgery may be used to reassess the disease depending on clinical judgment and patient willingness [5]. Second look evaluations were described by Wangensteen et al. [6] in 1951 as a way to prolong patient survival by removing early recurrences in patients with intra-abdominal malignancies. The initial aims of second look procedures were to establish an endpoint to chemotherapy cycles in patients with apparently complete clinical response, for secondary cytoreduction, and to direct subsequent treatment.

Due to recent use of serum tumor markers and advances in imaging technology such as ultrasound scan, MRI, CT scan, PET scan, and PET/CT scan, diagnosis of recurrent ovarian cancer has increased considerably [7]. However, these tests cannot diagnose small residual volume disease.

Until recently, the use of second look procedures was questioned due to lack of data suggesting survival advantage in patients with negative findings on second look. Due to the value of its prognostic information, ease of performance, and high patient acceptance, second look laparoscopy is a useful component of the standard treatment protocol for advanced ovarian cancer and as a primary laparoscopic debulking surgery for early ovarian cancer [8].

The purpose of our study is to compare the findings of investigative modalities and second look laparoscopy in ovarian cancer and to establish the safety and accuracy of second look laparoscopy for detecting ovarian cancer.

\section{Methods}

A retrospective review of all patients with ovarian cancer treated by a single surgeon at Ewha Women Cancer Center from 2006 to 2013 was performed. A total of 66 diagnosed ovarian cancer cases and 11 patients who had undergone second look laparoscopy were identified and included in the current study (16.66\%).

Of these patients, eight were diagnosed with stage III or IV disease at the time of primary cytoreductive surgery. All 11 patients had received six cycles of combination chemotherapy. These patients demonstrated complete clinical responses to primary chemotherapy. They were followed up with tumor markers monthly and PET-CT and/or CT scans. Clinical and pathologic review were performed for these patients, and institutional review board approval was granted by the local ethical committee (2018-06-041). The written patient consents were obtained.

\section{Second look laparoscopy}

All patients underwent second look laparoscopy 6 months after the primary operation. They were adequately informed and counseled regarding the procedure. Written informed consent was obtained. Second look laparoscopy was performed by using three ports. One $10 \mathrm{~mm}$ primary trocar was inserted by open technique. Two lateral trocars were $5 \mathrm{~mm}$.

The entire abdominal and pelvic cavities were inspected. Peritoneal washing was performed, and fluid was sent for cytology. Adhesiolysis was done if necessary. The area of primary disease was thoroughly inspected. Any suspicious areas were biopsied, multiple samples were taken from the anterior abdominal wall, anterior peritoneum, and pouch of Douglas and were sent for frozen section and histopathology evaluation.

\section{Tumor markers and imaging studies}

To compare the accuracy of investigative modalities, two site immunoenzymatic sandwich assay was used for CA 125, as per international standards. All values were determined in the same laboratory. The usual CA 125 cutoff for all clinical purposes is $35.0 \mathrm{U} / \mathrm{mL}$. CA 125 levels of all patients were analyzed at monthly intervals. The CA 125 value included in the study is the value determined up to 4 weeks before the second look laparoscopy.

PET-CT scan and CT/MRI scan were performed as per the protocol. PET-CT/CT scan was done by a dedicated Siemens Biograph mCT system. The minimum interval between chemotherapy and imaging study such as PET-CT scan was 6 weeks 
to minimize false negative findings that chemotherapy can cause by suppressing FDG uptake. The imaging studies were performed 6 months after the primary operation. All patients were required to fast before the studies, and basal sugars were determined to rule out hyperglycemia. Combined PET-CT and CT scans were performed.

\section{Results}

\section{Patient characteristics}

Of all 11 patients, two patients (patients 6 and 9) underwent primary laparotomy and the others underwent primary laparoscopy. Primary surgery was hysterectomy and bilateral salpingooophorectomy with cytoreductive surgery. Only one patient underwent unilateral oophorectomy with biopsy of the contralateral site for endometrioid carcinoma (patient 9). All second look surgery had undergone by laparoscopy. But one patient had been converted to laparotomy due to adhesion (patient 10). All patients had undergone primary surgery followed by six cycles of platinum-based chemotherapy. The median age of subjects was 54 years (range, 30 to 89 years). The median follow-up period was 54 months. The median difference between primary surgery and second look procedure was 8 months.

\section{Second look laparoscopy results}

Of 11 patients who underwent second look laparoscopy, eight patients $(72.7 \%)$ had positive pathologic findings and three patients $(27.3 \%)$ had negative pathologic findings (patients 7 to 9) (Table 1). The median number of biopsies taken was 7 (range, 1 to 20). Two patients underwent additional retroperitoneal lymphadenectomy on second look surgery (patients 3 and 10). The median hospital stay for second look laparoscopy was 10.45 days, and postoperative complications were not reported.

At the close of this study, the following findings were noted. Three patients with negative findings on tumor markers, imaging, and second look laparoscopy had no recurrence (5 years after second look laparoscopy) (patients 7 to 9). Seven patients (63.6\%) who had positive second look laparoscopy are surviving with the disease ( 5 years after second look laparoscopy) and were treated with consolidation chemotherapy (patients 2 to $6,10,11)$. And one patient $(12.5 \%)$ who had negative imaging study but positive tumor markers and second look laparoscopy succumbed to disease (patient 1).

\section{Tumor markers and imaging studies}

CA 125 level was higher in only one patient of eight patients with positive second look laparoscopy (12.5\%, patient 1$)$. The CA 125 level was determined at least 4 weeks before the second look laparoscopic procedure.

Four patients had a recorded increase in FDG uptake (36.4\%; patients $3,4,9,11)$. The increased standardized uptake value in specific regions of the scans of the three patients corresponded

Table 1. Second look findings for each patient with ovarian cancer

\begin{tabular}{|c|c|c|c|c|c|c|c|}
\hline Patient no. & Age (yr) & FIGO stage & Pathology & CA $125^{*}$ & CT scan & PET-CT & $\begin{array}{c}\text { Second look findgings } \\
\text { (positive) }\end{array}$ \\
\hline 1 & 82 & IV & Serous & 64.2 & - & - & Right pelvic \\
\hline 3 & 53 & IIIc & Serous & 8.0 & - & + & Right pelvic \\
\hline 4 & 51 & IV & Serous & 15.4 & - & + & Omentum \\
\hline 6 & 55 & Ic & Focal adeno squamous & 8.8 & - & - & Right pelvic \\
\hline 7 & 55 & IV & Transitional & 5.5 & - & - & - \\
\hline 8 & 69 & Ilc & Serous & 18.0 & - & - & - \\
\hline 9 & 31 & Ila & Endometrioid & 11.2 & - & + & - \\
\hline
\end{tabular}

FIGO, International Federation of Gynecology and Obstetrics; -, negative; +, positive.

${ }^{*}$ Cutoff value of CA 125 is $35.0 \mathrm{U} / \mathrm{mL}$. 


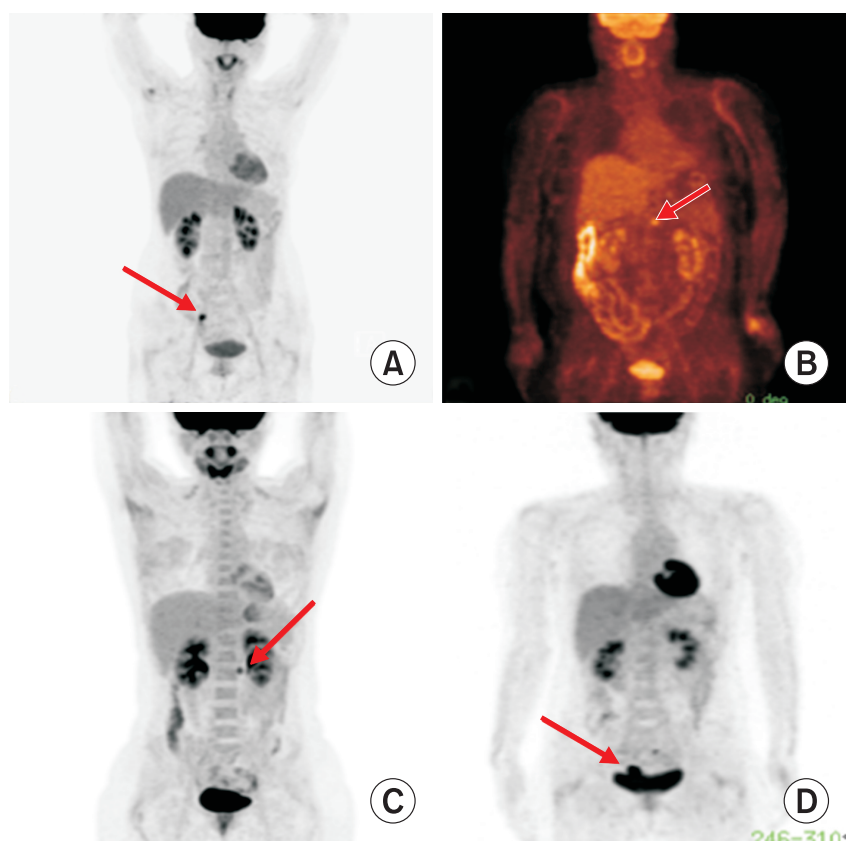

Fig. 1. Pre-second look laparoscopy PET-CT results. Arrows are indicating area of FDG uptakes. Increasing in FDG uptake on right periexternal vessel area (patient 3) (A), omental seeding (patient 4) (B), left paraspinal area of $\mathrm{L} 2$ spine, false positive (patient 9) (C), and right posterior side from bladder (patient 11) (D)

to positive biopsies from those regions (patients 3, 4, 11) (Fig. 1). The patient 9 had positive finding on PET-CT, but pathologic negative findings on second-look laparoscopy were interpreted as PET-CT uptake due to non-specific inflammation. The standardized uptake value cut off used in our institution is four. None of the patients had abnormal CT scan findings.

The sensitivity, specificity, positive predictive value and negative predictive value of the study were calculated based on the recurrence of cancer. And the recurrence was diagnosed by positive pathologic finding in second look laparoscopy. The negative predictive rates of PET-CT, CT scan, and tumor markers were $27.3 \%, 25.0 \%$, and $27.3 \%$, respectively (Table 2).

\section{Treatment after second look laparoscopy}

Eight patients had positive findings on second look laparoscopy (patients 1 to $6,10,11$ ). The seven patients were treated with consolidation chemotherapy and the other one died of advanced stage and disseminated intravascular coagulation (patient 1). Four patients were treated with topotecan-cisplatin chemotherapy, and two patients were treated with Taxotere-cisplatin
Table 2. Comparison of imaging study findings and second look findings in patients with ovarian cancer

\begin{tabular}{lcccc}
\hline & $\begin{array}{c}\text { Tumor } \\
\text { marker }\end{array}$ & CT scan & PET-CT & $\begin{array}{c}\text { Second } \\
\text { look } \\
\text { laparo- } \\
\text { scopy }\end{array}$ \\
\hline True positive & 1 & 0 & 3 & 8 \\
False positive & 0 & 0 & 1 & 0 \\
True negative & 3 & 3 & 2 & 3 \\
False negative & 7 & 8 & 5 & 0 \\
Sensitivity & 18.2 & 27.3 & 37.5 & 100 \\
Specificity & 100 & 100 & 66.7 & 100 \\
Positive predictive value & 100 & 100 & 75.0 & 100 \\
Negative predictive value & 27.3 & 25.0 & 28.6 & 100 \\
\hline
\end{tabular}

chemotherapy. One patient was treated with monotherapy topotecan regimen. Three patients with negative second look laparoscopy did not receive consolidation treatment (patients 7 to 9). Their disease-free survival were not calculated because of the recent nature of their disease.

The 5-year survival rate was $77.78 \%$ and it was calculated except two patients due to loss of following (patients 8 and 10). The 5-year survival rate for stage I was $100 \%$, stage II was $100 \%$, stage III was $66.67 \%$ and stage IV was $66.67 \%$. The 5 -year recurrence rate was $0 \%, 50 \%, 66.67 \%$, and $33.33 \%$ for stages I, II, III, and IV, respectively. The 5-year survival rate of patients with consolidation therapy was $66.67 \%$, and the 5-year recurrence rate was $33.33 \%$.

\section{Discussion}

The incidence of ovarian cancer has recently been rising, and diagnostic modalities have also been undergoing advancements [9]. Despite the availability of these advanced investigative modalities to diagnose recurrence, mortality caused by ovarian cancer appears to be increasing. This trend indicates that the available modalities are inconsistent in diagnosing small volume disease. In this context, we can consider using second look laparoscopy as a means to diagnose early recurrence.

The use of second look surgery has been debated [10]. Extensive laparotomy with multiple biopsies for second look has been associated with higher morbidity, blood loss, increased hospital stay, and lower patient compliance. Laparoscopy is a suitable second look modality because the visualization is better, 
and multiple biopsies, secondary cytoreduction, and retroperitoneal lymph node sampling can be achieved [8]. Minimally invasive surgery was introduced to surgical staging of ovarian cancer or debulking in early ovarian cancer because of benefits to patients. Therefore, second look laparoscopic surgery is available to patients who are expected to enter remission. After introducing robots in the gynecologic field, successful trials of primary or secondary robot-assisted laparoscopic surgeries were performed [11].

Other benefits of laparoscopic surgery include high patient acceptability, less operative time, lower morbidity, lower blood loss, and shorter hospital stay. While second look surgery failed to show survival benefit for patients with positive results, this result may be changing due to advances in high dose chemotherapy [12]. Recently, third look debulking surgery has been used to treat ovarian cancer [13]. The survival benefit of second look surgery remains contested. In our study, second look laparoscopic surgery was effective for diagnosing remnant ovarian cancer when negative predictive rates were low and all imaging studies were negative. Only 27.3\% of patients were diagnosed with persistent ovarian cancer by CT scan. Some studies also indicate that the risk of recurrence following negative second look laparoscopy is 50\% [14]. Indeed, many patients with advanced ovarian cancer eventually succumb to it, but the purpose of second look laparoscopy is to identify patients with no evidence of disease on other investigations who do have small volume disease and to treat them aggressively. This subset of patients would otherwise be missed if investigations such as tumor marker tests and imaging studies were the criteria for disease recurrence. Secondary cytoreduction carried out at the time of second look laparoscopy also improves the overall prognosis of these patients [15].

Hence, we must find a balance between the value of prognostic information obtained and the lack of demonstrated survival advantage in second look laparoscopy. Further consensus should be formed regarding the extent of surgery to be performed and the timing of surgery with respect to primary surgery and chemotherapy. A standard protocol for second look laparoscopy should be established for comparative study.

Our study compared the accuracy of the latest investigative modalities with findings of second look laparoscopy to diagnose recurrent ovarian cancer. There are limitations to the accuracy of investigative techniques and we must rely on clinical correlation between these modalities in each case with second look laparoscopic surgery. It is feasible to safely perform second look laparoscopic surgery to detect remnant ovarian cancer.

\section{References}

1. Ferlay J, Shin HR, Bray F, Forman D, Mathers C, Parkin DM. Estimates of worldwide burden of cancer in 2008: GLOBOCAN 2008. Int J Cancer 2010;127:2893-2917.

2. Jung KW, Won YJ, Kong HJ, Oh CM, Cho H, Lee DH, et al. Cancer statistics in Korea: incidence, mortality, survival, and prevalence in 2012. Cancer Res Treat 2015;47:127-141.

3. Park B, Park S, Kim TJ, Ma SH, Kim BG, Kim YM, et al. Epidemiological characteristics of ovarian cancer in Korea.J Gynecol Oncol 2010;21:241-247.

4. Zeng LJ, Xiang CL, Gong YZ, Kuang Y, Lu FF, Yi SY, et al. Neoadjuvant chemotherapy for patients with advanced epithelial ovarian cancer: a meta-analysis. Sci Rep 2016;6:35914.

5. Podratz KC, Kinney WK. Second-look operation in ovarian cancer. Cancer 1993;71:1551-1558.

6. Wangensteen OH, Lewis FJ, Tongen LA. The "second-look" in cancer surgery: a patient with colic cancer and involved lymph nodes negative on the "sixth-look". J Lancet 1951;71:303-307.

7. Hauth EA, Antoch G, Stattaus J, Kuehl H, Veit P, Bockisch A, et al. Evaluation of integrated whole-body PET/CT in the detection of recurrent ovarian cancer. Eur J Radiol 2005;56:263-268.

8. Husain A, Chi DS, Prasad M, Abu-Rustum N, Barakat RR, Brown $\mathrm{CL}$, et al. The role of laparoscopy in second-look evaluations for ovarian cancer. Gynecol Oncol 2001;80:44-47.

9. US Cancer Statistics Working Group. United States cancer statistics: 1999-2014 incidence and mortality web-based report [Internet]. Atlanta (GA): Department of Health and Human Services; Centers for Disease Control and Prevention; National Cancer Institute; c2017 [cited 2019 Feb 2]. Available from: http:// www.cdc.gov/uscs.

10. Friedman JB, Weiss NS. Second thoughts about secondlook laparotomy in advanced ovarian cancer. $N$ Engl J Med 1990;322:1079-1082.

11. Nezhat FR, Pejovic T, Finger TN, Khalil SS. Role of minimally invasive surgery in ovarian cancer. J Minim Invasive Gynecol 2013;20:754-765.

12. Schwartz PE, Smith JP. Second-look operations in ovarian cancer. Am J Obstet Gynecol 1980;138:1124-1130.

13. Dogan NU, Schneider A, Chiantera V, Dogan S, Dursun P. Tertiary cytoreduction in the setting of recurrent ovarian cancer (review). Oncol Lett 2013;6:642-647.

14. Rubin SC, Hoskins WJ, Saigo PE, Chapman D, Hakes TB, Markman M, et al. Prognostic factors for recurrence following negative second-look laparotomy in ovarian cancer patients treated with platinum-based chemotherapy. Gynecol Oncol 1991;42:137-141.

15. Oksefjell H, Sandstad B, Trope C. The role of secondary cytoreduction in the management of the first relapse in epithelial ovarian cancer. Ann Oncol 2009;20:286-293. 